\title{
IIMIII Assessing the Mental Health Needs and Barriers to Care Among a Diverse Sample of Asian American Older Adults
}

Dara H. Sorkin, $P h D^{7}$, Hannah Nguyen, MSW' , and Quyen Ngo-Metzger, MD, MPH ${ }^{7}$

'Division of General Internal Medicine and Primary Care and Health Policy Research Institute, University of California, Irvine, Irvine, CA, USA; ${ }^{2}$ Department of Social Welfare, University of California, Los Angeles, School of Public Affairs, Los Angeles, CA, USA.

BACKGROUND: Asian Americans represent a mix of cultures and immigration experiences, which may put them differentially at risk for mental health problems. Yet, little is known about the mental health needs of older adults from various Asian subgroups compared to non-Hispanic whites.

OBJECTIVES: To compare the prevalence rates of mental distress of Chinese, Filipino, South Asian, Japanese, Korean, and Vietnamese older adults (aged 55 and older) to that of non-Hispanic whites; and to examine subgroup differences in utilization of mental health services.

METHODS: A cross-sectional analysis of a populationbased sample of California adults responding to the 2007 California Health Interview Survey. Multivariable logistic regression analysis was used to examine subgroup differences in mental health status and use of mental health services among the six different Asian subgroups and non-Hispanic whites, adjusting for respondents' demographic and health characteristics, socioeconomic status, and English-language proficiency.

RESULTS: A total of 20,712 respondents were included. Filipino [aOR=2.25; 95\% CI=1.14-4.47] and Korean Americans $[\mathrm{aOR}=2.10 ; 95 \% \mathrm{CI}=1.06-4.17]$ were more likely to report symptoms indicative of mental distress compared to non-Hispanic whites, yet were less likely to have seen a primary care provider [Filipino: aOR $=0.41$; 95\% CI=0.18-0.90; Korean: $\mathrm{aOR}=0.24$; 95\% CI=0.080.69] or have taken a prescription medication [Filipino: aOR=0.20; 95\% CI=0.10-0.40; Korean: aOR=0.15; 95\% $\mathrm{CI}=0.05-0.40]$, even after adjusting for indicators of respondents' demographic and health characteristics, socioeconomic status, and English-language proficiency. In contrast, Japanese Americans were less likely to report symptoms indicative of mental distress [aOR=0.43; 95\% CI=0.21-0.90], and were less likely to make use of mental health services compared to nonHispanic whites.

DISCUSSION/CONCLUSIONS: The findings from this study not only highlight the unmet mental health needs among older Asian Americans, but also illustrate significant variations among the various Asian subgroups. Clinicians who work closely with these patients should regularly screen and assess older Asian adults for symptoms related to their mental health needs.

Received March 31, 2010

Revised August 26, 2010

Accepted December 7, 2010

Published online February 15, 2011
KEY WORDS: Asian Americans; mental health; aging; distress; California Health Interview Survey (CHIS).

J Gen Intern Med 26(6):595-602

DOI: $10.1007 / \mathrm{s} 11606-010-1612-6$

(C) The Author(s) 2011. This article is published with open access at Springerlink.com

\section{INTRODUCTION}

The Administration on Aging has estimated that between 2008 and 2030, the Asian American elderly population is projected to grow $199 \%$, to over 3.8 million people. ${ }^{1}$ As the number of Asian American elderly increases, so will their mental health needs. Estimates of the lifetime experience with depression, anxiety disorders, or substance abuse-related disorders for Asian Americans have ranged from $13.95 \%$ to as high as $18.29 \% .^{2,3}$ Limited research, however, has evaluated the mental health status of older Asian Americans. Sorkin, Pham, and Ngo-Metzger found, for example, that Asian American older adults were more likely to report mental distress compared to whites, even after adjusting for multiple sociodemographic characteristics and health status. ${ }^{4}$

Asian Americans represent over 20 ethnic subgroups, each with its distinct language, culture, educational level, and premigration and post-migration experience. ${ }^{5}$ In recognition of the marked heterogeneity of Asian Americans, an emerging body of research has examined differences in patterns of mental health status and use of mental health-related services at the individual ethnic subgroup level. ${ }^{2,6-8}$ For example, Shibusawa and Mui found that $20 \%$ of community-dwelling Japanese American elders were mildly depressed. ${ }^{9}$ Stokes et al. observed an even higher prevalence of depression among Chinese older adults at a rate of $29 \% .{ }^{10}$

The high prevalence of distress among older Asian Americans is likely to result, in part, from factors related to their immigration experiences. For example, many older immigrants have difficulty finding work in the US and encounter related financial difficulties. ${ }^{11}$ Lacking insurance and not being proficient in English may limit one's access to health services, ${ }^{12-14}$ which, in turn, may further negatively impact the mental health of older immigrant adults. Language barriers also may be especially important in mental health care settings, given the heavy reliance on direct verbal communication to express symptoms and to understand health information and treatment. ${ }^{15}$

In spite of the documented need, studies have consistently found lower use of mental health services among Asian 
Americans compared with the general population. ${ }^{4,6,16}$ While research has pointed to patterns of mental health needs and service underutilization of Asian Americans, most of these studies are based on convenience samples, and few have examined subgroup differences. ${ }^{17}$ The aims of our study were to examine subgroup differences in the mental health status and use of mental health services of Asian American older adults. Using the 2007 California Health Interview Survey (CHIS), we examined the prevalence rates of self-reported symptoms indicative of mental distress among Chinese, Filipino, South Asian, Japanese, Korean, and Vietnamese older adults $(\geq 55)$ living in California compared to nonHispanic whites. We also examined differences in the use of mental health services (including having seen a primary care provider, another professional, or having taken a prescription medication) during the past 12 months across the subgroups. We hypothesized that Asian American older adults would be more likely to have mental health problems, but less likely to utilize specialty mental health services compared to non-Hispanic whites. We also hypothesized that there would be significant variations in the mental health needs and use of services among the Asian subgroups compared to non-Hispanic whites. To our knowledge, this investigation is among the first studies to examine factors associated with mental health service use in a population-based sample that includes six different Asian subgroups.

\section{METHODS}

\section{Procedure}

The 2007 California Health Information Survey (CHIS) is a biannual, random-digit dial telephone survey designed to be representative of the Californian non-institutionalized population. One randomly-selected adult per household was sampled between July 2007 and March 2008. ${ }^{18}$ Interviews were conducted in five languages, based on 2000 Census data of the languages spoken most often by Californian residents. For non-English speaking individuals, the survey was administered in Spanish, Chinese (Mandarin and Cantonese), Vietnamese, or Korean. Approximately 647 surveys were administered in a language besides English. To ensure crosscultural equivalence across items, the survey underwent refereed translation processes and extensive cultural adaptation. Additional information about the cultural translation and sampling methodology can be found elsewhere. ${ }^{18,19}$

\section{Sample}

The data set used for this study comes from the CHIS Public Use File and Confidential Data 2007. The CHIS employs a twostage geographically stratified random-digit-dial sample design to collect data from every county in California, which after weighting provides an unbiased representation of the California population. The sample is weighted to be representative of California's population in terms of age, sex, race/ethnicity, and rural-urban residence. ${ }^{18}$

The sample for these analyses was restricted to adults, aged 55 years and older, who reported their race/ethnicity as either
non-Hispanic white or Asian American. The specific Asian ethnic backgrounds of the participants assessed included: 496 Chinese, 255 Filipinos, 87 South Asians, 263 Japanese, 288 Koreans, and 175 Vietnamese. An additional 42 respondents were identified as either Cambodian, other Asian, or multiple Asian, but were not included in the current sample because of their small sample size. More information about the survey can be obtained from the California Health Interview Survey website: http://www.chis.ucla.edu/.

\section{Measures \\ Outcomes}

Psychological Distress. Psychological distress was assessed with the Kessler 6 (K6) Scale. ${ }^{20,21}$ The K6 scale asks respondents about the extent to which they experienced six different manifestations of psychological distress over the past 30 days: (a) so depressed that nothing could cheer you up, (b) nervous, (c) hopeless, (d) restless or fidgety, (e) worthless, and (f) everything was an effort. Ratings were made on a 5-point scale ranging from 0 (none of the time) to 4 (all of the time). Items were summed to create a composite measure, ranging from 0 to 24. According to scoring criterion established by Kessler, a score of $\geq 6$ indicates non-specific psychological mental distress. ${ }^{20,22,23}$

Use of Mental Health Services. Mental health service use was assessed by asking all respondents if they saw either their primary care physician/general practitioner or any other professional, such as a counselor, psychiatrist or social worker for problems with mental health or substance abuse. All respondents were asked whether they took any prescription medications, such as an antidepressant or sedative almost daily or for two weeks or more, for their emotional or personal problem during the past 12 months. Possible answers to each question were Yes/No.

Covariates. Standard demographic characteristics were assessed, such as age, gender $(1=$ male, $2=$ female $)$, nativity ( 1 = born in the US, 2 = foreign born), and marital status $(1=$ not currently married, 2 = currently married). Physical health status was assessed by asking participants whether they had been diagnosed with any of four chronic health conditions (i.e., high blood pressure, asthma, diabetes mellitus, and heart disease). Responses were summed to form a measure of number of chronic health conditions $(0=$ None, $1=1$ or 2 chronic conditions, $2=3$ or 4 chronic conditions). Indicators of respondents' socioeconomic status were assessed, including education ( 1 = completed high school or less, $2=$ some college or more), employment status ( 1 = currently employed, $2=$ not currently employed, but looking for employment, and $3=$ not currently employed), and health insurance status $(1=$ uninsured or not continuously insured, 2 = continuous insurance over past 12 months). In addition, food security was measured by asking respondents with a household income less than or equal to $200 \%$ of Federal Poverty Level (FPL) how often it was true that they experienced periods during the year when they could not afford to put food on the 
table or had to forgo other basic needs to do so, and whether or not these low-income, food-insecure adults experienced episodes of hunger. In 2007, the federal poverty guideline for a single, elderly person was an annual income of $\$ 10,210$, and for an older couple, $\$ 13,690$, which is well below the basic annual cost of living for a retired older adult in California, which averages $\$ 21,011 .^{24}$ Responses where recoded into three categories: $1=$ food security ( $>200 \% \mathrm{FPL}$ ), $2=$ food security ( $\leq 200 \% \mathrm{FPL})$, and 3 = food insecurity $(\leq 200 \% \mathrm{FPL}$, with and without hunger). English language proficiency was dichotomized into two categories, according to self-reported responses to the question "Would you say you speak English..." (1 = Very well/Well, 2 = Not well/Not at all).

\section{Statistical Analyses}

SAS Callable SUDAAN Release 9.0.2 (Research Triangle Institute, Research Triangle Park, NC) was used to conduct analyses to account for the complex sampling design, as well as to use weighted effects for accurate variance estimates. Data analysis was carried out in three phases. First, descriptive statistics were generated examining the socio-demographic and general health characteristics of the study sample. To examine differences in the socio-demographic characteristics by subgroup, bi-variable analyses were conducted using Chi-square tests to compare categorical variables and ANOVA-tests for continuous variables. Second, one way ANOVAs were conducted to examine subgroup differences in mental health status and utilization. Finally, we conducted multivariable logistic regression models to determine the relationship between ethnicity and each of the outcomes (i.e., mental health status and utilization). A priori, we included in the adjusted models as covariates other variables that have been linked to ethnic differences in mental health status and use of services. Specifically, we built three models for each outcome, adding groups of variables in a sequential manner as follows: 1) ethnicity and demographic and health information, including age, gender, marital status, and physical health status; 2) indicators of socioeconomic status (i.e., insurance status, education, employment status, and food insecurity); and 3) English-language proficiency. Two-tailed $\mathrm{P}$ values less than 0.05 were considered statistically significant. To account for the multiple comparisons, we used a Bonferroni correction to adjust the $\mathrm{P}$ value to be less than or equal to 0.008 and indicated in Tables 3, 4, 5, 6 results that remained significant.

\section{RESULTS}

Table 1 presents the sociodemographic and health characteristics of the study sample. Ninety to $100 \%$ of respondents in the Asian sub-groups were born outside of the US, except for the Japanese (32\%) who were more likely to be born in the US. Most to all respondents had resided in the US for 15 years or more at the time of the survey. More than half of all respondents across all subgroups reported having one to two chronic health conditions. Educational attainment varied considerably among Asian Americans. Educational attainment was the highest among South Asians (87.2\%) and the lowest among the Vietnamese (24.9\%). Korean and Vietnamese older adults had the highest rates of being uninsured, and Vietnamese $(37.1 \%)$ older adults reported the highest rates of food insecurity. The majority of Korean (70.3\%), Chinese $(51.8 \%)$, and Vietnamese $(84.1 \%)$ respondents reported being limited English-language proficient compared to Filipino (7.2\%), Japanese (6.1\%), and South Asian (3.5\%) respondents.

Unadjusted differences in mental health status and utilization of mental health services by race and ethnicity are presented in Table 2. As shown, Filipino (20.3\%), Korean (18.0\%) and Chinese (12.0\%) older adults were significantly more likely than non-Hispanic whites $(9.7 \%)$ to report symptoms indicative of mental distress $(\underline{P}<0.001)$. Korean, Japanese, and Chinese older adults also were significantly less likely to see their primary care physician or another mental health professional compared to non-Hispanic whites. Finally, respondents from all Asian sub-groups were less likely to take a prescription medication for mental health compared to nonHispanic white respondents.

Tables 3, 4, 5, 6 present the adjusted models, evaluating subgroup differences in mental health status and use of mental health services among the six different Asian subgroups and non-Hispanic whites, controlling for respondents' demographic and health characteristics, socioeconomic status, and English-language proficiency. As shown in model 3 of Table 3, Filipino Americans had more than two times the odds of reporting symptoms indicative of mental distress compared to non-Hispanic whites [aOR=2.25; 95\% CI=1.14-4.47], even after adjusting for indicators of demographic, health, socioeconomic status, and English-language proficiency. Yet, they were less likely to have seen a primary care provider [aOR=0.41;95\% CI=0.18-0.90] or have taken a prescription medication $[\mathrm{aOR}=0.20 ; 95 \% \mathrm{CI}=0.10-0.40]$, as shown in model 3 of Tables 4 and 6 , respectively.

Similarly, Korean Americans also were more likely to report symptoms indicative of mental distress compared to non-Hispanic whites [See model 3, Table 3: aOR=2.10; 95\% $\mathrm{CI}=1.06-4.17]$ and were less likely to make use of any mental health services, including seeing a primary care provider or other professional or taking a prescription medication (See Tables 4, 5, 6: aORs range=0.15 to 0.24). The difference between Koreans and non-Hispanic whites in the odds of having seen a professional [aOR=0.34; 95\% $\mathrm{CI}=0.10-1.18]$ was attenuated, however, when English-language proficiency was included in the model (see model 3, Table 5).

Japanese respondents were less likely to report symptoms indicative of mental distress compared to non-Hispanic whites [See model 3, Table 3: aOR=0.43; 95\% CI=0.21-0.90], even after adjusting for indicators of demographic and health characteristics socioeconomic status, and English-language proficiency, and also were less likely than non-Hispanic whites to report making use of any mental health services (see Tables 4, 5, 6: aORs range $=0.16$ to 0.18 ).

\section{DISCUSSION}

This study is among the first to compare differences in the mental health status and utilization of mental health services among six Asian American subgroups to non-Hispanic white older adults using a large, ethnically and racially diverse 
Table 1. Sociodemographic and Health Status Differences by Race/Ethnicity ${ }^{\S}$

\begin{tabular}{|c|c|c|c|c|c|c|c|c|}
\hline & $\begin{array}{l}\text { Filipino } \\
(n=258)\end{array}$ & $\begin{array}{l}\text { Korean } \\
(n=288)\end{array}$ & $\begin{array}{l}\text { Japanese } \\
(n=268)\end{array}$ & $\begin{array}{l}\text { Chinese } \\
(n=496)\end{array}$ & $\begin{array}{l}\text { Vietnamese } \\
(n=175)\end{array}$ & $\begin{array}{l}\text { South Asian } \\
(n=87)\end{array}$ & $\begin{array}{l}\text { non-Hispanic } \\
\text { white } \\
(n=19,098)\end{array}$ & $p$-value \\
\hline Age & $65.7 \mathrm{yrs}$ & $66.1 \mathrm{yrs}$ & $71.3 \mathrm{yrs}$ & $67.8 \mathrm{yrs}$ & $64.6 \mathrm{yrs}$ & 65.9 yrs & $67.7 \mathrm{yrs}$ & $<0.001$ \\
\hline \multicolumn{9}{|l|}{ Gender } \\
\hline Male $(n=8,067)$ & $41.6 \%$ & $44.1 \%$ & $33.1 \%$ & $43.9 \%$ & $52.9 \%$ & $68.0 \%$ & $46.3 \%$ & \multirow[t]{2}{*}{$<0.001$} \\
\hline Female $(n=12,645)$ & $58.4 \%$ & $55.9 \%$ & $66.9 \%$ & $56.2 \%$ & $47.1 \%$ & $32.0 \%$ & $53.8 \%$ & \\
\hline \multicolumn{9}{|l|}{ Marital Status } \\
\hline Not currently married $(n=10,448)$ & $27.0 \%$ & $24.9 \%$ & $33.3 \%$ & $23.7 \%$ & $22.9 \%$ & $12.5 \%$ & $35.9 \%$ & \multirow[t]{2}{*}{$<0.001$} \\
\hline Currently married $(n=10,264)$ & $73.0 \%$ & $75.1 \%$ & $66.7 \%$ & $76.3 \%$ & $77.1 \%$ & $87.5 \%$ & $64.2 \%$ & \\
\hline \multicolumn{9}{|l|}{ Born in the United States } \\
\hline Yes $(n=18,005)$ & $6.5 \%$ & $0.7 \%$ & $67.8 \%$ & $10.5 \%$ & $0 \%$ & $0 \%$ & $91.7 \%$ & \multirow[t]{2}{*}{$<0.001$} \\
\hline No $(n=27,076)$ & $93.5 \%$ & $99.3 \%$ & $32.2 \%$ & $89.5 \%$ & $100 \%$ & $100 \%$ & $8.3 \%$ & \\
\hline \multicolumn{9}{|l|}{ Years in the United States ${ }^{\ddagger}$} \\
\hline Less than $15(n=129)$ & $13.2 \%$ & $12.5 \%$ & $0 \%$ & $21.7 \%$ & $32.6 \%$ & $22.9 \%$ & $3.1 \%$ & \multirow[t]{2}{*}{$<0.001$} \\
\hline 15 or more $(n=2,578)$ & $86.8 \%$ & $87.5 \%$ & $100 \%$ & $78.3 \%$ & $67.4 \%$ & $77.1 \%$ & $96.9 \%$ & \\
\hline \multicolumn{9}{|l|}{ Number of chronic health conditions } \\
\hline $0(\mathrm{n}=7,577)$ & $21.9 \%$ & $44.0 \%$ & $37.2 \%$ & $38.2 \%$ & $33.5 \%$ & $29.3 \%$ & $37.5 \%$ & \multirow[t]{3}{*}{$<0.001$} \\
\hline $1-2(n=11,500)$ & $67.2 \%$ & $54.4 \%$ & $58.2 \%$ & $57.1 \%$ & $56.9 \%$ & $61.4 \%$ & $54.4 \%$ & \\
\hline $3-4(n=1,635)$ & $10.9 \%$ & $1.6 \%$ & $4.6 \%$ & $4.7 \%$ & $9.6 \%$ & $9.3 \%$ & $8.1 \%$ & \\
\hline \multicolumn{9}{|l|}{ Type of chronic health condition } \\
\hline High blood pressure $(n=10,501)$ & $68.6 \%$ & $46.1 \%$ & $55.6 \%$ & $47.8 \%$ & $55.4 \%$ & $60.3 \%$ & $49.6 \%$ & \multirow[t]{4}{*}{$<0.001$} \\
\hline Diabetes $(n=11,500)$ & $17.4 \%$ & $18.7 \%$ & $15.0 \%$ & $15.5 \%$ & $19.8 \%$ & $28.5 \%$ & $13.3 \%$ & \\
\hline Asthma $(n=2,697)$ & $13.2 \%$ & $4.3 \%$ & $8.8 \%$ & $7.4 \%$ & $6.9 \%$ & $9.5 \%$ & $13.0 \%$ & \\
\hline Heart disease $(n=3,678)$ & $18.5 \%$ & $6.6 \%$ & $9.7 \%$ & $13.1 \%$ & $15.5 \%$ & $19.0 \%$ & $17.8 \%$ & \\
\hline \multicolumn{9}{|l|}{ Insurance status } \\
\hline Continuously insured $(n=19,756)$ & $96.0 \%$ & $76.4 \%$ & $96.6 \%$ & $91.8 \%$ & $86.1 \%$ & $89.5 \%$ & $95.6 \%$ & \multirow[t]{2}{*}{$<0.001$} \\
\hline $\begin{array}{l}\text { Uninsured or not continuously } \\
\text { insured }(n=956)\end{array}$ & $4.1 \%$ & $23.6 \%$ & $3.5 \%$ & $8.2 \%$ & $13.9 \%$ & $10.5 \%$ & $4.4 \%$ & \\
\hline \multicolumn{9}{|l|}{ Education } \\
\hline High school degree or less $(n=5,693)$ & $25.8 \%$ & $49.1 \%$ & $37.7 \%$ & $43.5 \%$ & $75.1 \%$ & $12.8 \%$ & $34.2 \%$ & \multirow[t]{2}{*}{$<0.001$} \\
\hline $\begin{array}{l}\text { Some college degree or more } \\
(n=15,019)\end{array}$ & $74.2 \%$ & $51.0 \%$ & $62.3 \%$ & $56.5 \%$ & $24.9 \%$ & $87.2 \%$ & $65.8 \%$ & \\
\hline \multicolumn{9}{|l|}{ Current employment status } \\
\hline Currently employed $(n=7,130)$ & $49.5 \%$ & $41.7 \%$ & $25.0 \%$ & $30.3 \%$ & $23.8 \%$ & $56.8 \%$ & $39.4 \%$ & \multirow[t]{3}{*}{$<0.001$} \\
\hline Not currently employed $(n=12,517)$ & $48.3 \%$ & $55.4 \%$ & $74.0 \%$ & $66.5 \%$ & $69.8 \%$ & $43.2 \%$ & $58.4 \%$ & \\
\hline $\begin{array}{l}\text { Not employed, but looking for job } \\
(\mathrm{n}=413)\end{array}$ & $2.2 \%$ & $2.9 \%$ & $1.0 \%$ & $3.2 \%$ & $6.4 \%$ & $0.0 \%$ & $2.3 \%$ & \\
\hline \multicolumn{9}{|l|}{ Poverty level and food security status } \\
\hline $\begin{array}{l}>200 \% \text { FPL, Food security } \\
(n=16,519)\end{array}$ & $70.7 \%$ & $49.4 \%$ & $87.3 \%$ & $55.6 \%$ & $21.7 \%$ & $64.6 \%$ & $83.8 \%$ & \multirow[t]{3}{*}{$<0.001$} \\
\hline $\begin{array}{l}\leq 200 \% \text { FPL, Food security } \\
(n=3,373)\end{array}$ & $23.3 \%$ & $43.0 \%$ & $12.0 \%$ & $34.2 \%$ & $41.2 \%$ & $33.9 \%$ & $13.1 \%$ & \\
\hline$\leq 200 \%$ FPL, Food insecurity $(n=778)$ & $6.0 \%$ & $7.6 \%$ & $0.7 \%$ & $10.2 \%$ & $37.1 \%$ & $1.5 \%$ & $3.1 \%$ & \\
\hline \multicolumn{9}{|l|}{ English language proficiency } \\
\hline Very well/Well $(n=20,050)$ & $92.8 \%$ & $29.7 \%$ & $93.9 \%$ & $48.2 \%$ & $15.9 \%$ & $96.5 \%$ & $99.4 \%$ & \multirow[t]{2}{*}{$<0.001$} \\
\hline Not well/Not at all $(n=662)$ & $7.2 \%$ & $70.3 \%$ & $6.1 \%$ & $51.8 \%$ & $84.1 \%$ & $3.5 \%$ & $0.6 \%$ & \\
\hline
\end{tabular}

¥ This question was only asked of those respondents born outside of the United States

${ }^{\S}$ Percentages are weighted

Table 2. Differences in Mental Health Status and Utilization by Race/Ethnicity

\begin{tabular}{|c|c|c|c|c|c|c|c|c|}
\hline & $\begin{array}{l}\text { Filipino } \\
(n=255)\end{array}$ & $\begin{array}{l}\text { Korean } \\
(n=288)\end{array}$ & $\begin{array}{l}\text { Japanese } \\
(n=263)\end{array}$ & $\begin{array}{l}\text { Chinese } \\
(n=496)\end{array}$ & $\begin{array}{l}\text { Vietnamese } \\
(n=175)\end{array}$ & $\begin{array}{l}\text { South Asian } \\
(n=87)\end{array}$ & $\begin{array}{l}\text { Non-Hispanic } \\
\text { white }(n=19,098)\end{array}$ & $p$-value \\
\hline $\begin{array}{l}\text { Reported symptoms indicative of } \\
\text { mental distress }(n=20,712)\end{array}$ & $20.3 \% *$ & $18.0 \% *$ & $3.6 \% *$ & $12.0 \%$ & $14.2 \%$ & $12.7 \%$ & $9.7 \%$ & $<0.001$ \\
\hline $\begin{array}{l}\text { Saw a primary care physician or } \\
\text { general practitioner for problems } \\
\text { with mental health or substance } \\
\text { abuse }(n=20,568)\end{array}$ & $3.0 \%$ & $1.6 \% *$ & $1.0 \% *$ & $1.0 \% *$ & $4.9 \%$ & $1.7 \%$ & $6.5 \%$ & $<0.001$ \\
\hline $\begin{array}{l}\text { Saw other professional } \\
\text { (i.e., counselor, psychiatrist, } \\
\text { social worker) for problems } \\
\text { with mental health or } \\
\text { substance abuse }(\mathrm{n}=20,568)\end{array}$ & $6.7 \%$ & $2.2 \%{ }^{*}$ & $0.9 \%{ }^{*}$ & $2.2 \%{ }^{*}$ & $3.2 \%{ }^{*}$ & $2.4 \%$ & $7.2 \%$ & $<0.001$ \\
\hline $\begin{array}{l}\text { Took a prescription medication } \\
\text { for mental health }(n=20,568)\end{array}$ & $3.8 \%{ }^{*}$ & $2.7 \%{ }^{*}$ & $2.5 \%{ }^{*}$ & $4.1 \%{ }^{*}$ & $9.2 \%^{*}$ & $4.7 \%{ }^{*}$ & $14.0 \%$ & $<0.001$ \\
\hline
\end{tabular}

§Percentages are weighted

* Significantly different from non-Hispanic white, $P<0.05$ 
Table 3. Comparing Asian to non-Hispanic White (NHW) Older Adults in Reported Symptoms Indicative of Mental Distress

\begin{tabular}{|c|c|c|c|}
\hline & Model 1 & Model 2 & Model 3 \\
\hline & OR $(95 \% \mathrm{Cl})$ & OR $(95 \% \mathrm{Cl})$ & OR $(95 \% \mathrm{Cl})$ \\
\hline \multicolumn{4}{|l|}{ Ethnicity $($ Ref $=$ NHW) } \\
\hline Filipino & $2.22(1.21-4.06)^{*}$ & $2.20(1.10-4.37)^{*}$ & $2.25(1.14-4.47)^{*}$ \\
\hline Korean & $2.25(1.31-3.86)^{*^{+}}$ & $1.62(0.90-2.91)$ & $2.10(1.06-4.17)^{*}$ \\
\hline Japanese & $0.40(0.20-0.81)^{*}$ & $0.42(0.20-0.87)^{*}$ & $0.43(0.21-0.90)^{*}$ \\
\hline Chinese & $1.42(0.97-2.07)$ & $1.07(0.72-1.58)$ & $1.35(0.83-2.18)$ \\
\hline Vietnamese & $1.53(0.91-2.58)$ & $0.58(0.32-1.04)$ & $0.81(0.40-1.61)$ \\
\hline South Asian & $1.47(0.85-2.54)$ & $1.63(0.94-2.84)$ & $1.65(0.95-2.88)$ \\
\hline Age & $0.96(0.95-0.97)^{*}$ & $0.95(0.94-0.97)^{*}$ & $0.96(0.94-0.97)^{*}$ \\
\hline Female $($ Ref $=$ male $)$ & 1.34 (1.09-1.64)* & $1.26(1.01-1.56)^{*}$ & $1.26(1.01-1.57)^{*}$ \\
\hline Married (Ref = not currently married) & $0.61(0.50-0.74)^{*}$ & $0.75(0.61-0.92)^{*}$ & $0.76(0.62-0.93)^{*}$ \\
\hline \multicolumn{4}{|l|}{ Physical Health Status (Ref = none) } \\
\hline 1-2 Chronic conditions & $1.38(1.13-1.67)^{*}$ & $1.31(1.06-1.62)^{*}$ & $1.31(1.06-1.62)^{*}$ \\
\hline 3-4 Chronic conditions & $3.06(2.29-4.09)^{*}$ & $2.37(1.72-3.27)^{*}$ & $2.36(1.71-3.25)^{*}$ \\
\hline Insured (Ref = uninsured) & & $0.71(0.49-1.01)$ & $0.70(0.49-0.99)^{*}$ \\
\hline Some college or more (Ref $=\leq$ high school) & & $0.90(0.73-1.11)$ & $0.89(0.72-1.09)$ \\
\hline \multicolumn{4}{|l|}{ Employment status (Ref = not employed) } \\
\hline Currently employed & & $0.61(0.46-0.80)^{*}$ & $0.61(0.46-0.80)^{*}$ \\
\hline Not employed, but looking for job & & $1.22(0.79-1.90)$ & $1.23(0.79-1.91)$ \\
\hline \multicolumn{4}{|l|}{ Food security $(\mathrm{Ref}=>200 \% \mathrm{FPL})$} \\
\hline$\leq 200 \%$ FPL, food security & & $1.84(1.46-2.32)^{*}$ & $1.88(1.40-2.37)^{*}$ \\
\hline$\leq 200 \% \mathrm{FPL}$, food insecurity & & $4.79(3.57-6.43)^{*}$ & $4.93(3.68-6.62)^{*}$ \\
\hline English language proficiency (Ref = very well/well)) & & & $0.80(0.46-1.37)$ \\
\hline Wald F & 209.68 & 173.51 & 169.30 \\
\hline df & 12 & 18 & 19 \\
\hline$\Delta$ Wald F Sig. & & $<0.001$ & $<0.005$ \\
\hline
\end{tabular}

Note: The Wald F statistics was used to assess model fit because the approximate Chi-Square (-2 * Log-L Ratio) does not adjust for clustering

${ }^{+} p \leq 0.008$. Reflects the critical value for the test statistic after applying Bonferroni correction for multiple comparisons

$* p \leq 0.05$

Table 4. Comparing Asian to non-Hispanic White (NHW) Older Adults in Reported Visit to a Primary Care Provider

\begin{tabular}{|c|c|c|c|}
\hline & Model 1 & Model 2 & Model 3 \\
\hline & OR $(95 \% \mathrm{Cl})$ & OR $(95 \% \mathrm{Cl})$ & OR $(95 \% \mathrm{Cl})$ \\
\hline \multicolumn{4}{|l|}{ Ethnicity $($ Ref $=$ NHW $)$} \\
\hline Filipino & $0.37(0.16-0.87)^{*}$ & $0.39(0.18-0.89)^{*}$ & $0.41(0.18-0.90)^{*}$ \\
\hline Korean & $0.24(0.08-0.69)^{*+}$ & $0.15(0.06-0.42) *^{+}$ & $0.24(0.08-0.69)^{*}$ \\
\hline Japanese & $0.17(0.06-0.47)^{*+}$ & $0.18(0.06-0.49) *^{+}$ & $0.18(0.07-0.50) *^{*+}$ \\
\hline Chinese & $0.16(0.08-0.33)^{*+}$ & $0.16(0.08-0.32) *^{+}$ & $0.22(0.10-0.50) *^{*+}$ \\
\hline Vietnamese & $0.69(0.32-1.47)$ & $0.43(0.19-0.99)^{*}$ & $0.80(0.22-2.90)$ \\
\hline South Asian & $0.28(0.02-4.09)$ & $0.33(0.02-4.61)$ & $0.34(0.02-4.79)$ \\
\hline Age & $0.94(0.93-0.95)^{*}$ & $0.92(0.91-0.94)^{*}$ & $0.92(0.91-0.94)^{*}$ \\
\hline Female $($ Ref $=$ male $)$ & $1.95(1.59-2.40)^{*}$ & $1.83(1.50-2.23)^{*}$ & $1.83(1.50-2.22)^{*}$ \\
\hline Married (Ref = not currently married) & $0.59(0.46-0.75)^{*}$ & $0.63(0.49-0.82)^{*}$ & $0.64(0.49-0.83)^{*}$ \\
\hline \multicolumn{4}{|l|}{ Physical health status (Ref = none) } \\
\hline 1-2 Chronic conditions & $1.58(1.19-2.09)^{*}$ & $1.45(1.09-1.92)^{*}$ & $1.44(1.09-1.92)^{*}$ \\
\hline 3-4 Chronic conditions & $3.37(2.23-5.10)^{*}$ & $2.61(1.67-4.07)^{*}$ & $2.60(1.67-4.04)^{*}$ \\
\hline Insured (Ref = uninsured) & & $0.88(0.60-1.29)$ & $0.88(0.60-1.28)$ \\
\hline Some college or more (Ref $=\leq$ high school) & & $1.09(0.89-1.33)$ & $1.07(0.86-1.32)$ \\
\hline \multicolumn{4}{|l|}{ Employment status (Ref = not employed) } \\
\hline Currently employed & & $0.46(0.34-0.63)^{*}$ & $0.46(0.34-0.63)^{*}$ \\
\hline Not employed, but looking for job & & $0.84(0.49-1.42)$ & $0.84(0.49-1.42)$ \\
\hline \multicolumn{4}{|l|}{ Food security $(\mathrm{Ref}=>200 \% \mathrm{FPL})$} \\
\hline$\leq 200 \% \mathrm{FPL}$, food security & & $1.21(0.92-1.59)$ & $1.23(0.94-1.61)$ \\
\hline$\leq 200 \%$ FPL, food insecurity & & $2.03(1.43-2.86)^{*}$ & $2.06(1.46-2.92)^{*}$ \\
\hline English language proficiency $($ Ref = very well/well) & & & $0.48(0.16-1.46)$ \\
\hline Wald F & 312.67 & 237.95 & 228.21 \\
\hline df & 12 & 18 & 19 \\
\hline$\Delta$ Wald F Sig. & & $<0.001$ & $<0.001$ \\
\hline
\end{tabular}

Note: The Wald F statistics was used to assess model fit because the approximate Chi-Square (-2* Log-L Ratio) does not adjust for clustering

${ }^{+} p \leq 0.008$. Reflects the critical value for the test statistic after applying Bonferroni correction for multiple comparisons

$* p \leq 0.05$ 
Table 5. Comparing Asian to non-Hispanic White (NHW) Older Adults in Reported Visit to a Mental Health Care Professional

\begin{tabular}{|c|c|c|c|}
\hline & Model 1 & Model 2 & Model 3 \\
\hline & OR $(95 \% \mathrm{Cl})$ & OR $(95 \% \mathrm{Cl})$ & OR $(95 \% \mathrm{Cl})$ \\
\hline \multicolumn{4}{|l|}{ Ethnicity $($ Ref $=$ NHW $)$} \\
\hline Filipino & $0.83(0.38-1.82)$ & $0.83(0.39-1.77)$ & $0.85(0.40-1.82)$ \\
\hline Korean & $0.31(0.11-0.86)^{*}$ & $0.26(0.08-0.80)^{*}$ & $0.34(0.10-1.18)$ \\
\hline Japanese & $0.16(0.05-0.50) *^{+}$ & $0.16(0.05-0.50) *^{*+}$ & $0.16(0.05-0.51)^{*+}$ \\
\hline Chinese & $0.32(0.16-0.67) *^{+}$ & $0.35(0.17-0.72) *^{+}$ & $0.42(0.20-0.90)^{*}$ \\
\hline Vietnamese & $0.39(0.18-0.85)^{*}$ & $0.32(0.14-0.75)^{*}$ & $0.46(0.19-1.15)$ \\
\hline South Asian & $0.35(0.11-1.19)$ & $0.37(0.11-1.22)$ & $0.37(0.11-1.25)$ \\
\hline Age & $0.92(0.91-0.94)^{*}$ & $0.91(0.90-0.93)^{*}$ & $0.91(0.90-0.93)^{*}$ \\
\hline Female (Ref $=$ male $)$ & $1.43(1.20-1.70)^{*}$ & $1.37(1.14-1.65)^{*}$ & $1.37(1.14-1.65)^{*}$ \\
\hline Married (Ref = not currently married) & $0.51(0.42-0.62)^{*}$ & $0.53(0.43-0.65)^{*}$ & $0.53(0.43-0.66)^{*}$ \\
\hline \multicolumn{4}{|l|}{ Physical health status (Ref = none) } \\
\hline 1-2 Chronic conditions & $1.20(0.97-1.49)$ & $1.13(0.91-1.41)$ & $1.13(0.91-1.40)$ \\
\hline 3-4 Chronic conditions & $1.88(1.24-2.84)^{*}$ & $1.64(1.07-2.49)^{*}$ & $1.63(1.06-2.49)^{*}$ \\
\hline Insured (Ref = uninsured) & & $1.34(0.80-2.24)$ & $1.34(0.80-2.24)$ \\
\hline Some college or more (Ref $=\leq$ high school) & & $1.59(1.22-2.06)^{*}$ & $1.57(1.21-2.05)^{*}$ \\
\hline \multicolumn{4}{|l|}{ Employment status $($ Ref $=$ not employed $)$} \\
\hline Currently employed & & $0.61(0.49-0.77)^{*}$ & $0.61(0.49-0.77)^{*}$ \\
\hline Not employed, but looking for job & & $0.92(0.59-1.43)$ & $0.92(0.60-1.43)$ \\
\hline \multicolumn{4}{|l|}{ Food security $(\mathrm{Ref}=>200 \% \mathrm{FPL})$} \\
\hline$\leq 200 \% \mathrm{FPL}$, food security & & $1.33(0.97-1.84)$ & $1.36(0.99-1.87)$ \\
\hline$\leq 200 \%$ FPL, food insecurity & & $1.63(1.12-2.38)^{*}$ & $1.65(1.13-2.42)^{*}$ \\
\hline English language proficiency (Ref = very well/well) & & & $0.62(0.27-1.42)$ \\
\hline Wald F & 433.17 & 278.77 & 265.53 \\
\hline df & 12 & 18 & 19 \\
\hline$\Delta$ Wald F Sig. & & $<0.001$ & $<0.001$ \\
\hline
\end{tabular}

Note: The Wald F statistics was used to assess model fit because the approximate Chi-Square (-2 * Log-L Ratio) does not adjust for clustering

${ }^{+} p \leq 0.008$. Reflects the critical value for the test statistic after applying Bonferroni correction for multiple comparisons

$* p \leq 0.05$

Table 6. Comparing Asian to non-Hispanic white (NHW) Older Adults in Reported Use of a Prescription Medication

\begin{tabular}{|c|c|c|c|}
\hline & Model 1 & Model 2 & Model 3 \\
\hline & OR $(95 \% \mathrm{Cl})$ & OR $(95 \% \mathrm{Cl})$ & OR $(95 \% \mathrm{Cl})$ \\
\hline \multicolumn{4}{|l|}{ Ethnicity $($ Ref $=$ NHW $)$} \\
\hline Filipino & $0.19(0.09-0.40) *^{*+}$ & $0.20(0.10-0.40) *^{+}$ & $0.20(0.10-0.40){ }^{*+}$ \\
\hline Korean & $0.17(0.07-0.41) *^{+}$ & $0.18(0.07-0.44) *^{+}$ & $0.15(0.05-0.40) *^{+}$ \\
\hline Japanese & $0.17(0.08-0.35)^{*+}$ & $0.17(0.08-0.35){ }^{*+}$ & $0.17(0.08-0.35))^{*+}$ \\
\hline Chinese & $0.27(0.17-0.41)^{*+}$ & $0.28(0.18-0.43){ }^{*+}$ & $0.24(0.15-0.37)^{*+}$ \\
\hline Vietnamese & $0.57(0.34-0.95)^{*}$ & $0.42(0.25-0.71) *^{+}$ & $0.33(0.19-0.59) *^{+}$ \\
\hline South Asian & $0.32(0.10-1.04)$ & $0.37(0.11-1.21)$ & $0.37(0.11-1.19)$ \\
\hline Age & $0.95(0.94-0.96)^{*}$ & $0.94(0.93-0.95)^{*}$ & $0.94(0.93-0.95)^{*}$ \\
\hline Female $($ Ref $=$ male $)$ & $2.10(1.81-2.45)^{*}$ & $1.98(1.72-2.29)^{*}$ & $1.98(1.71-2.29)^{*}$ \\
\hline Married (Ref = not currently married) & $0.71(0.60-0.83)^{*}$ & $0.71(0.60-0.84)^{*}$ & $0.70(0.60-0.83)^{*}$ \\
\hline \multicolumn{4}{|l|}{ Physical health status (Ref = none) } \\
\hline 1-2 Chronic conditions & $1.62(1.38-1.91)^{*}$ & $1.55(1.31-1.83)^{*}$ & $1.50(1.31-1.83)^{*}$ \\
\hline 3-4 Chronic conditions & $3.06(2.39-3.91)^{*}$ & $2.59(1.98-3.39)^{*}$ & $2.60(1.99-3.40)^{*}$ \\
\hline Insured (Ref=uninsured) & & $1.35(0.99-1.84)$ & $1.35(0.98-1.84)$ \\
\hline Some college or more (Ref $=\leq$ high school) & & $1.13(0.96-1.33)$ & $1.13(0.96-1.34)$ \\
\hline \multicolumn{4}{|l|}{ Employment status $($ Ref $=$ not employed $)$} \\
\hline Currently employed & & $0.53(0.43-0.66)^{*}$ & $0.53(0.43-0.66)^{*}$ \\
\hline Not employed, but looking for job & & $0.63(0.43-0.92)^{*}$ & $0.63(0.42-0.92)^{*}$ \\
\hline \multicolumn{4}{|l|}{ Food security $(\mathrm{Ref}=>200 \% \mathrm{FPL})$} \\
\hline$\leq 200 \% \mathrm{FPL}$, food security & & $1.03(0.84-1.26)$ & $1.02(0.83-1.25)$ \\
\hline$\leq 200 \%$ FPL, food insecurity & & $1.74(1.26-2.42)^{*}$ & $1.72(1.23-2.41)^{*}$ \\
\hline English language proficiency (Ref = very well/well) & & & $1.33(0.87-2.03)$ \\
\hline Wald $\mathrm{F}$ & 436.30 & 341.60 & 355.54 \\
\hline df & 12 & 18 & 19 \\
\hline$\Delta$ Wald F Sig. & & $<0.001$ & n.s. \\
\hline
\end{tabular}

Note: The Wald F statistics was used to assess model fit because the approximate Chi-Square (-2 * Log-L Ratio) does not adjust for clustering

${ }^{+} p \leq 0.008$. Reflects the critical value for the test statistic after applying Bonferroni correction for multiple comparisons

$* p \leq 0.05$ 
population-based sample. In this representative cohort of California's non-institutionalized older adults, Filipino and Korean older adults were more likely to report symptoms indicative of mental distress compared to non-Hispanic whites. Yet, the findings suggested that they were less likely to see a primary care provider or take any prescription medications for an emotional or personal problem compared to non-Hispanic white older adults.

Although there are 2.9 million Filipinos living in the US, there is a relative dearth of literature on the mental health status of Filipino Americans. ${ }^{25,26}$ Filipino Americans are among the earliest US immigrants. ${ }^{27}$ While the Filipino older adults in this sample were much more likely to speak English and be well-educated compared to other Asian subgroups, they, nonetheless, may have encountered immigration stress. ${ }^{28}$ The poor economy in the Philippines has led many Filipino professionals (including nurses, doctors, and engineers) to leave their families and immigrate to the US. Often their professional degrees are not recognized in the US, leading to under- or unemployment and decreased social status. Role strain is often reported by Filipino professionals, ${ }^{11}$ who must split their lives between the US and families back home and between their own culture and US culture. As such, previous research has found that among Filipinos diagnosed with clinical depression, separation from family and financial difficulties are two of the most commonly reported stressors. ${ }^{25}$

The high rate of mental distress among Korean American older adults also is consistent with previous research. ${ }^{29-31}$ Although nearly all of the Korean respondents in this sample were born in Korea, most immigrated to the US more than 15 years ago. Despite this, over 70\% reported that they spoke English not well or not at all. Furthermore, nearly one-quarter of older Koreans in this sample reported being uninsured. The high rates of poor English proficiency coupled with the lack of insurance may leave older Koreans vulnerable. ${ }^{31}$ Interestingly, among Koreans, there is a recognition of high rates of symptom manifestation similar to major depressive disorder in the Korean folk illness labeled 'Hwa-byung'. ${ }^{32}$ Hwa-byung is characterized by somatic and psychological symptoms, such as compression in the chest, loss of appetite and sleep, and excessive anxiety. ${ }^{33}$ Researchers have found a higher prevalence of Hwa-byung among Koreans living in the US than among those living in Korea, suggesting that this syndrome may be a culturally appropriate way for individuals to express their distress when adjusting to their lives in the US. ${ }^{32}$

The finding that Japanese older adults reported less distress than their non-Hispanic white counterparts differs from those found in two other studies, which found moderate to high prevalence of depression among Japanese elders. ${ }^{13,34}$ These studies, however, involved either a convenience sample ${ }^{34}$ or had a very small sample size $(n=25),{ }^{13}$ and neither study compared older Japanese with older whites. The Japanese respondents in our study were more likely to be born in the US and perhaps may not have experienced the level of migration and acculturative stress that other Asian immigrants faced. ${ }^{35}$ Furthermore, the socioeconomic standing of the Japanese older adults in this sample looked very similar to or exceeded that of the nonHispanics whites, and thus, might explain their relatively better mental health.

Despite some of the Asian American subgroups having higher rates of mental distress, older adults of all Asian American subgroups (except South Asians) were less likely to make use of mental health services compared to non-Hispanic white older adults. Although some Asian Americans reported seeking help for their mental health problems, many continued to underutilize care. ${ }^{6,36,37}$ Structural barriers, such as a lack of awareness of available services, lack of insurance, and language barriers, may make it difficult for older adults to get the care they need. ${ }^{6,16,37}$ Cultural barriers, such as stigma and denial, have been linked to low mental health service use. ${ }^{38}$ Culturally responsive services that take into account the unique cultural and religious values, ${ }^{8,39}$ including the importance of the family, and pre- and post-migration experience, are needed. ${ }^{40}$ Furthermore, clinicians need to be open to considering alternative or unconventional treatments, as in nearly all contemporary Asian societies, multiple healing traditions exist that thrive side by side with conventional Western health care systems. ${ }^{40}$

Several limitations of the current study should be noted. First, because CHIS is a population-based sample drawn from the civilian, non-institutionalized adult population in California living in households with access to a residential telephone, individuals without residential telephones or those living in group quarters of unrelated adults (a common situation among recent immigrants with limited income) may be excluded from the study. The findings, therefore, likely underestimate the mental health needs of older Asian American adults who are frail, in poor health, or are more recent immigrants. In addition, while data for the current is drawn from a representative sample of older adults living in California, which has some of the highest proportions of these Asian American subgroups in the country, ${ }^{5}$ these findings may not generalize to older Asian adults in other areas of the United States.

Nonetheless, this study adds to the scant literature on the mental health needs and access to care among Asian American older adults. The findings from this study not only highlight the unmet mental health needs among older Asian Americans, but also illustrate differences among the various Asian subgroups. Taken together, these findings imply that medical and social service professionals who work closely with these groups should regularly screen and assess older adults' depressive symptoms. ${ }^{41}$ Efforts to increase the rate at which patients with significant psychological symptoms are identified and referred to mental health services by their primary care provider will help address the unmet mental health needs of ethnically/racially diverse older adults. ${ }^{42}$

Acknowledgments: Financial Disclosure: The authors have no financial disclosures and no conflict of interests to disclose related to this manuscript. Dr. Sorkin received support from a NIH KOI award (NIDDK DK 078939). Dr. Ngo-Metzger received support from the Robert Wood Johnson Foundation, Generalist Physician Faculty Scholar Award (Grant \#1051084).

\section{Conflict of Interest: None disclosed.}

Open Access: This article is distributed under the terms of the Creative Commons Attribution Noncommercial License which permits any noncommercial use, distribution, and reproduction in any medium, provided the original author(s) and source are credited.

Corresponding Author: Dara H. Sorkin, PhD; Division of General Internal Medicine and Primary Care and Health Policy Research Institute, University of California, Irvine, Irvine, CA, USA (e-mail:dsorkin@uci.edu). 


\section{REFERENCES}

1. A Statistical Profile of Asian Older Americans Aged 65 and Older. http:// www.aoa.gov/AoARoot/Aging_Statistics/Minority_Aging/Facts-on-APIElderly2008-plain_format.aspx. Accessed December 7, 2010.

2. Takeuchi DT, Zane $\mathbf{N}$, Hong S, et al. Immigration-related factors and mental disorders among Asian Americans. Am J Public Health. 2007;97 (1):84-90.

3. Takeuchi DT, Chung RC, Lin K-M, et al. Lifetime and twelve-month prevalence rates of major depressive episodes and dysthymia among Chinese Americans in Los Angeles. Am J Psychiatry. 1998;155 (10): 1407-1414.

4. Sorkin DH, Pham E, Ngo-Metzger Q. Racial and ethnic differences in the mental health needs and access to care of older adults in California. JAGS. 2009;57:2311-2317.

5. Trinh-Shevrin C, Islam NS, Rey MJ. Asian American Communities and Health: Context, Research, Policy, and Action. San Francisco: Jossey-Bass.

6. Abe-Kim J, Takeuchi DT, Hong S, et al. Use of Mental Health-Related Services Among Immigrant and US-Born Asian Americans: Results From the National Latino and Asian American Study. Am J Public Health. 2007;97(1):91-98.

7. Breslau J, Chang DF. Psychiatric disorders among foreign-born and USborn Asian Americans in a US national survey. Soc Psychiatry Psychiatr Epidemiol. 2006;41:943-950.

8. Ta VM, Holck P, Gee GC. Generational status and family cohesion effects on the receipt of mental health services among Asian Americans: findings from the National Latino and Asian American Study. Am J Public Health. 2010;100(1):115-121.

9. Shibusawa T, Mui AC. Stress, coping and depression among Japanese American elders. J Gerontol Soc Work. 2002;26(1/2):63-82.

10. Stokes SC, Thompson LW, Murphy S, Gallagher-Thompson D. Screening for depression in immigrant Chinese-American elders: Results of a pilot study. J Gerontol Soc Work. 2002;36(1/2):27-44.

11. Root MPP. Filipino Families. In: McGoldrick M, Giordano J, Garcia-Preto N, eds. Ethnicity \& Family Therapy. 3rd ed. New York: Guilford Press; 2005.

12. Kim G, Aguado Loi CX, Chiriboga DA, et al. Limited English proficiency as a barrier to mental health service use: A study of Latino and Asian immigrants with psychiatric disorders. J Psychiatr Res. May 252010.

13. Mui AC, Kang SY. Acculturation stress and depression among Asian immigrant elders. Soc Work. 2006;51(3):243-255.

14. Sorkin DH, Ngo-Metzger Q, De Alba I. Racial/ethnic discrimination in healthcare: Impact on quality of care. J Gen Intern Med. 2010.

15. Sentell T, Shumway M, Snowden L. Access to mental health treatment by English language proficiency and race/ethnicity. J Gen Intern Med. 2007;22(Suppl 2):289-293.

16. Leong FT, Lau AS. Barriers to providing effective mental health services to Asian Americans. Ment Health Serv Res. 2001;3(4):201-214.

17. Herrick CA, Brown HN. Underutilization of mental health services by Asian-Americans residing in the United States. Issues Ment Health Nurs. 1998; 19(3):225-240.

18. California Heath Interview Survey. CHIS Survey Methodology and Sample Design. http://www.chis.ucla.edu/methods_main.html [web page]. 2005. Accessed December 7, 2010.

19. Ponce N, Lavarreda SA, Yen W, et al. The California Health Interview Survey 2001: Translation of a major survey for California's multiethnic population. Public Health Rep. 2004;119(4):388-395.

20. Kessler RC, Andrews G, Colpe LJ, et al. Short screening scales to monitor population prevalences and trends in non-specific psychological distress. Psychol Med. 2002;32:959-976.

21. Kessler RC, Barker PR, Colpe LJ, et al. Screening for serious mental illness in the general population. Arch Gen Psychiatry. 2003;60:184189.
22. Andrews G, Slade T. Interpreting scores on the Kessler Psychological Distress Scale (K10). Aust New Zeal J Publ Health. 2001;25(6):494497.

23. Fukukawa TA, Kessler RC, Slade T, Andrews G. The performance of the K6 and K10 screening scales for psychological distress in the Australian National Survey of Mental Health and Well-Being. Psychol Med. 2003;33:357-362.

24. Wallace SP, Molina LC. Federal poverty guideline underestimates costs of living for older persons in California. UCLA Health Policy Research Brief. Feb 2008. http://escholarship.org/uc/item/Ofb3f5dg;jsessionid=16D9449B71243CB78AF1BBEBC41128F2. Accessed December 7, 2010.

25. Sanchez F, Gaw A. Mental health care of Filipino Americans. Psychiatr Serv. 2007;58(6):810-815.

26. Zhang W, Ta VM. Social connections, immigration-related factors, selfrated physical and mental health among Asian Americans. Soc Sci Med. 2009;68:2104-2112.

27. Boyd M. Oriental Immigration: The Experience of the Chinese, Japanese, and Filipino Populations in the United States. Int Migrat Rev. 1971;5(1):48-61.

28. Brown DE, James GD. Physiological stress responses in FilipinoAmerican immigrant nurses: the effects of residence time, life-style, and job strain. Psychosom Med. 2000;62(3):394-400.

29. Mui AC. Stress, coping and depression among elderly Korean immigrants. J Hum Behav Soc Environ. 2001;3(3/4):281-299.

30. Sohn L. The health and health status of older Korean Americans at the 100-year anniversary of Korean immigration. J Cross Cult Gerontol. 2004; 19(3):203-219.

31. Mui AC, Kang SY, Kang D, Domanski MD. English language proficiency and health-related quality of life among Chinese and Korean immigrant elders. Health Soc Work. 2007;32(2):119-127.

32. Lin KM, Lau J, Yamamoto $\mathbf{J}$, et al. Hwa-byung: A community study of Korean Americans. J Nerv Ment Dis. 1992;180(6):386-391.

33. Lin KM. Hwa-Byung: a Korean culture-bound syndrome? Am J Psychiatr. 1983;140:105-107.

34. Gellis ZD, Taguchi A. Depression and health status among communitydwelling Japanese American elderly. Clin Gerontologist. 2004;27 (3):23-38.

35. Shibusawa T, Chung I. The Health of the Elderly. In: Trinh-Shevrin C, Islam NS, Rey MJ, eds. Asian American Communities and Health: Context, Research, Policy, and Action. San Francisco: Wiley; 2009: 199-225.

36. Meyer OL, Zane N, Cho YI, Takeuchi D. Use of specialty mental health services by Asian Americans with psychiatric disorders. J Consult Clin Psychol. 2009;77(5):1000-1005.

37. Zhang AY, Snowden LR, Sue S. Differences between Asian and white Americans' help seeking and utilization patterns in the Los Angeles area. J Community Psychol. 1998;26(4):317-326.

38. Kim G, Jang Y, Chiriboga DA, Ma GX, Schonfeld L. Factors associated with mental health service use in Latino and Asian immigrant elders. Aging Ment Health. 2010;14(5):535-542.

39. Gary FA. Stigma: barrier to mental health care among ethnic minorities. Issues Ment Health Nurs. 2005;26:979-999.

40. Lin KM, Cheung F. Mental health issues for Asian Americans. Psychiatr Serv. 1999;50(6):774-780.

41. Kuo BC, Chong V, Joseph J. Depression and its psychosocial correlates among older Asian immigrants in North America: a critical review of two decades' research. J Aging Health. 2008;20(6):615-652.

42. Wong EC, Schell TL, Marshall GN, Jaycox LH, Hambarsoomians $\mathbf{K}$, Belzberg $\mathbf{H}$. Mental health service utilization after physical trauma: the importance of physician referral. Med Care. 2009;47 (10): 1077-1083. 